\section{(1) \\ CrossMark}

\title{
BNP/NT-proBNP in pulmonary arterial hypertension: time for point-of-care testing?
}

\author{
Robert A. Lewis ${ }^{1,2}$, Charlotte Durrington ${ }^{1}$, Robin Condliffe ${ }^{1}$ and David G. Kiely (1) ${ }^{1,2,3}$ \\ Affiliations: ${ }^{1}$ Sheffield Pulmonary Vascular Disease Unit, Royal Hallamshire Hospital, Sheffield, UK. ${ }^{2}$ Dept of \\ Infection, Immunity and Cardiovascular Disease, University of Sheffield, Sheffield, UK. ${ }^{3}$ Insigneo Institute for \\ in silico medicine, University of Sheffield, Sheffield, UK.
}

Correspondence: David G. Kiely, Sheffield Pulmonary Vascular Disease Unit, Room M15, Ward M2, Royal Hallamshire Hospital, Sheffield, S10 2JF, UK. E-mail: david.kiely1anhs.net

@ERSpublications

Despite the widespread use of BNP and NT-proBNP in pulmonary arterial hypertension there are limited data on the standardisation of sampling and no data on the use of point-of-care testing and its potential value in clinical management https://bit.ly/2Xxm9uV

Cite this article as: Lewis RA, Durrington C, Condliffe R, et al. BNP/NT-proBNP in pulmonary arterial hypertension: time for point-of-care testing? Eur Respir Rev 2020; 29: 200009 [https://doi.org/10.1183/ 16000617.0009-2020].

ABSTRACT Despite the advent of new therapies and improved outcomes in patients with pulmonary arterial hypertension (PAH), it remains a life-shortening disease and the time to diagnosis remains unchanged. Strategies to improve outcomes are therefore currently focused on earlier diagnosis and a treatment approach aimed at moving patients with $\mathrm{PAH}$ into a category of low-risk of 1-year mortality. B-type natriuretic peptide (BNP; or brain natriuretic peptide) and N-terminal prohormone of BNP (NTproBNP) are released from cardiac myocytes in response to mechanical load and wall stress. Elevated levels of BNP and NT-proBNP are incorporated into several PAH risk stratification tools and screening algorithms to aid diagnosis of systemic sclerosis. We have undertaken a systematic review of the literature with respect to the use of BNP and NT-proBNP in PAH and the use of these biomarkers in the diagnosis and risk stratification of $\mathrm{PAH}$, their relation to pulmonary haemodynamics and the potential for point-of-care testing to improve diagnosis and prognosis.

\section{Introduction}

Pulmonary arterial hypertension (PAH), or World Health Organization (WHO) Group 1 pulmonary hypertension $(\mathrm{PH})$, is a rare, progressive condition characterised by endothelial dysfunction and vascular remodelling, leading to increases in mean pulmonary artery pressure (mPAP), pulmonary vascular resistance (PVR) and ultimately right ventricular (RV) failure. PAH may occur sporadically (idiopathic PAH) but may also be drug induced or associated with other conditions, such as connective tissue disease, congenital heart disease, portal hypertension or HIV infection [1-3]. Current guidelines require patients to have a right heart catheterisation (RHC) measured $\mathrm{mPAP} \geqslant 25 \mathrm{mmHg}$, pulmonary artery wedge pressure $\leqslant 15 \mathrm{mmHg}$ and a PVR of $>3$ WU at rest, in the absence of other causes [1]. Recently, the $6^{\text {th }}$ World Symposium on Pulmonary Hypertension Task Force proposed a new definition of mPAP $>20 \mathrm{mmHg}$ and PVR $\geqslant 3$ WU [4].

Symptoms and survival are primarily related to the development of RV dysfunction and include breathlessness, fatigue and, in severe disease, fluid retention and syncope $[5,6]$. The estimated prevalence of PAH reported by national registries ranges between 10 and 52 cases per million worldwide [7].

Provenance: Publication of this peer-reviewed article was sponsored by Janssen Ltd, UK (article sponsor, European Respiratory Review issue 156).

Received: 13 Jan 2020 | Accepted after revision: 06 April 2020

Copyright $\odot$ ERS 2020. This article is open access and distributed under the terms of the Creative Commons Attribution Non-Commercial Licence 4.0. 
Over the past two decades, there have been significant improvements in patient outcomes [8]. Treatment of PAH primarily encompasses pharmacological and lifestyle interventions [1]. Current pharmacological therapies target three distinct biological pathways (prostanoid, endothelin and nitric oxide), with evidence for superiority of combination therapy over monotherapy [1]. The aim of therapy is to maintain or achieve a low-risk profile, thus establishing the severity of disease and serially assessing response to therapy is key to informed decision making.

Current approaches to risk stratification rely on assessments of symptoms, exercise capacity and RV function. There are a number of risk stratification scores, including REVEAL 2.0 [9] and scores developed by European registries based on the European Society of Cardiology/European Respiratory Society (ESC/ ERS) recommendations $[1,10]$.

For assessment of RV function, cardiac magnetic resonance (CMR) imaging is the gold standard [11-13], but cost, availability and until recently the paucity of data on its value in risk stratification [13] have limited its use. Active B-type natriuretic peptide (BNP; or brain natriuretic peptide) and the functionally inert N-terminal prohormone of BNP (NT-proBNP) are well-established clinical biomarkers used in PAH and other cardiovascular disorders, such as acute/chronic heart failure and are used as surrogate markers of cardiac function $[1,14,15]$. NT-proBNP is also used to screen for PAH in patients with systemic sclerosis (SSc) [16]. Typically, BNP or NT-proBNP are measured when patients are assessed by their PAH physician and this information is integrated with the results of other investigations. Typically, clinical laboratory-based assays using venous blood samples are used, and results are not immediately available. In contrast, point-of-care testing (POCT) devices can be used to ensure that results are immediately available and can inform decision making at the time of the consultation. In this review we summarise the current evidence for the use of BNP and NT-proBNP in the early diagnosis of PAH and risk stratification, and explore the potential for POCT in PAH.

\section{Physiology of natriuretic peptides and the role of BNP and NT-proBNP in cardiovascular disease and PAH}

Natriuretic peptides are a family of hormones secreted primarily from the heart, kidneys and brain that cause vasodilation and natriuresis. They include atrial natriuretic peptide, BNP, C-type natriuretic peptide and urodilatin. Atrial natriuretic peptide has a short half-life of $\sim 2 \mathrm{~min}$ and is sensitive to temperature change [17]. In contrast, BNP has a longer half-life of $\sim 22 \mathrm{~min}$ and is more stable, making it more attractive as a clinical biomarker [17]. In a study examining the stability of atrial natriuretic peptide and BNP, blood samples were shown to be stable ex vivo for $2 \mathrm{~h}$ and 2 days, respectively [18], though some deterioration of BNP can be observed within $4 \mathrm{~h}$ at room temperature [19].

BNP is a product of the early-response gene $N P P B$. It is mainly synthesised de novo and secreted by the ventricular myocardium in response to mechanical, hormonal or sympathetic stimulation, with levels in blood peaking $\sim 1 \mathrm{~h}$ after stimulation [20]. In PAH, transmural pressure, volume overload, hypoxia or pro-inflammatory factors induce transcription of $N P P B$ to produce 134 -amino acid (aa) preproBNP. A signal peptide is subsequently removed in the sarcoplasmic reticulum, leaving 108 -aa proBNP. This is then cleaved on secretion into the bloodstream to produce the two biomarkers of 32 -aa BNP and 76-aa NT-proBNP (figure 1) [14, 21-23]. BNP binds to the natriuretic peptide receptor-A, which is primarily expressed in kidney, adrenal, lung, terminal ileum, aorta and adipose tissue [14]. Receptor activation leads to increases in the intracellular secondary messenger cyclic guanosine monophosphate, resulting in vasodilation, natriuresis, aldosterone inhibition and lipolysis [14]. NT-proBNP has no known function but due to its longer half-life compared with BNP (70 min versus $22 \mathrm{~min}$ ) [14] and relative stability in storage [19], it has potential handling advantages as a biomarker.

\section{BNP/NT-proBNP and comparison with pulmonary haemodynamics, echocardiographic and CMR metrics}

It is well established that BNP and NT-proBNP correlate with a number of pulmonary haemodynamic metrics that are associated with survival. Utilising RHC, CHIN et al. [24] showed that BNP correlates with right atrial pressure (RAP; $\mathrm{r}=0.66$ ) in patients with PAH. In SSc-PAH, Williams et al. [25] demonstrated that NT-proBNP correlated with mPAP $(r=0.62)$, PVR $(r=0.81)$, RAP $(r=0.53)$ and cardiac index $(r=$ -0.50 ), and also found NT-proBNP to be an independent predictor of survival. In a study of 30 patients undergoing RHC and CMR at baseline and follow-up, changes in NT-proBNP correlated with changes in RAP ( $r=0.49)$, cardiac index $(r=-0.45)$, PVR index $(r-0.30)$, RV end-diastolic volume index $(r=0.59)$, $\mathrm{RV}$ mass index ( $\mathrm{r}=0.62$ ) and right ventricular ejection fraction (RVEF; $r=-0.81$ ) [26].

Similar findings have been reported using echocardiographic techniques. In chronic heart failure, TROISI et al. [27] found BNP levels to be correlated with TAPSE ( $\mathrm{r}-=0.33)$, RAP $(\mathrm{r}=0.34)$ and PAP ( $\mathrm{r}=0.42)$. In PAH, Gото et al. [28] reported correlations between BNP levels and transthoracic echocardiography (TTE)-measured systolic PAP when left ventricular (LV) dysfunction was excluded ( $\mathrm{r}=0.51)$. 


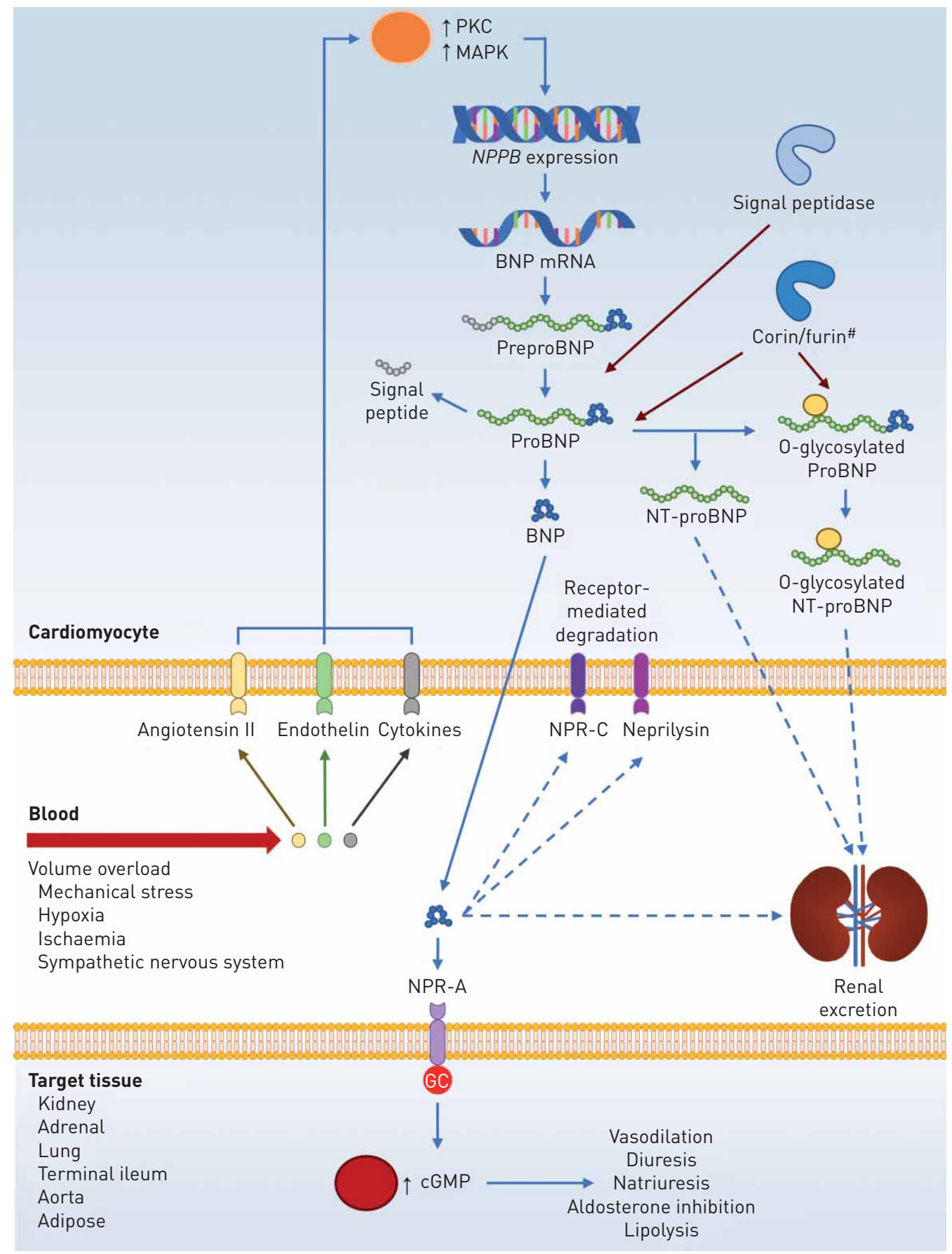

FIGURE 1 Molecular pathways of synthesis and release of B-type natriuretic peptide (BNP) and N-terminal prohormone of BNP (NT-proBNP). aa: amino acid; cGMP: cyclic guanosine monophosphate; GC: guanylyl cyclase; MAPK: mitogen-activated protein kinase; NPR: natriuretic peptide receptor; PKC: protein kinase C. \#: candidate enzymes which may produce 29 aa or 32aa BNP depending on cleavage site $[22,23]$.

In a recent study focused on patients with WHO functional class (FC) III PAH who underwent blood testing for BNP, RHC and TTE, BNP levels had positive correlations with RHC-measured RAP ( $\mathrm{r}=0.20$ ), mPAP ( $\mathrm{r}=0.25)$ and PVR $(\mathrm{r}=0.31)$, and negative correlations with cardiac output $(\mathrm{r}=-0.33)$ and cardiac index $(r=-0.28)$ [29]. Using TTE, BNP positively correlated with right atrial $(r=0.18)$ and $R V$ enlargement $(\mathrm{r}=0.17)$, and RV dysfunction ( $\mathrm{r}=0.15)$. In addition, BNP levels correlated with lower 6-min walk distance (6MWD; $\mathrm{r}=-0.75)$ and higher WHO FC ( $\mathrm{r}=0.26)$.

Levels of BNP and NT-proBNP have also been shown to correlate with CMR measures of RV structure and function in small studies. In PAH, a cohort of 14 patients ( $\mathrm{n}=12$ idiopathic PAH) found Spearman's 
coefficients of correlation with RVEF for BNP and NT-proBNP of -0.54 and -0.61 , respectively [30]. Similar correlations have been reported by BLYTH et al. [31] in PAH and chronic thromboembolic $\mathrm{PH}$ $(\mathrm{n}=25)$, who reported a significant association of NT-proBNP and CMR-RVEF $(\mathrm{r}=-0.66)$. This study also reported a sensitivity and specificity of NT-proBNP for detecting RV systolic dysfunction, defined as CMR-derived RVEF $>2$ SD below controls of $100 \%$ and $94 \%$, respectively. In other disorders, a study including 21 patients with minimally symptomatic congenital heart disease-related chronic RV pressure overload found BNP levels to be inversely correlated with RVEF ( $r=-0.65)$ [32].

\section{Risk stratification in PAH and the role of BNP/NT-proBNP}

Current approaches to risk stratification use a multiparameter approach. The 2015 ESC/ERS guidelines do not explicitly function as a risk calculator, but give reference values for multiple risk parameters that clinicians can interpret in the clinical context [1]. Several studies have validated these ESC/ERS parameters at baseline and follow-up assessment within different European populations [2, 33-36]. These studies have used differing adaptations to the ESC/ERS approach to account for the fact that it is possible for parameters within a single patient to fall into different risk groups.

Boucly et al. [35] examined a large cohort of patients with idiopathic PAH $(n=1017)$ and developed a risk stratification score incorporating WHO/New York Heart Association FC, 6MWD, RAP and CI, quantifying the number of low-risk criteria to predict transplant-free survival in the French Pulmonary Hypertension Registry. Furthermore, they identified that replacing RAP and cardiac index with BNP/ NT-proBNP in a subgroup $(n=603)$ could also discriminate prognostic groups, an approach which has the potential to reduce the requirement for haemodynamic evaluation. Patients achieving all three low-risk criteria (WHO/ New York Heart Association FC I or II, $6 \mathrm{MWD}>440 \mathrm{~m}$, and $\mathrm{BNP}<50 \mathrm{ng} \cdot \mathrm{L}^{-1}$ or NT-proBNP $<300 \mathrm{ng} \cdot \mathrm{L}^{-1}$ ) had 2-, 3- and 5-year survival of $100 \%$, 99\% and $97 \%$, respectively. This method has since been validated in data from the Phase 3 GRIPHON trial, which reported a $94 \%$ reduced risk of morbidity/mortality in patients with all three low-risk criteria versus those with no low-risk values [37].

Further validation for the ESC/ERS approach has been demonstrated in the Comparative, Prospective Registry of Newly Initiated Therapies for Pulmonary Hypertension (COMPERA), which was able to achieve accurate mortality estimates by using an adapted version of the guidelines [2]. By grading each measure to give each patient a single composite risk score, the study demonstrated 1-year mortality rates of $2.8 \%, 9.9 \%$ and $21.2 \%$ in low-, intermediate- and high-risk groups, respectively.

In contrast to the ESC/ERS guidelines, REVEAL assigns an overall score based on multiple demographic, haemodynamic and serum parameters in order to establish risk of 1-year mortality, and has also been validated in multiple studies [9, 38-41]. An update, REVEAL 2.0, has recently been published [9] which, in an independent cohort, demonstrated 1-year mortality estimates of $2.6 \%, 8.6 \%$ and $25.4 \%$ for low-, intermediate- and high-risk groups respectively, with 5-year estimates of $16.1 \%, 41.5 \%$ and $88.0 \%$, respectively [41]. Additionally, a study using CMR of RV end systolic volume index adjusted for age and sex has been shown to further improve risk stratification for 1-year mortality when used with either the REVEAL 2.0 score or a modified French Pulmonary Hypertension Risk score [13].

An additional factor when considering the clinical practicality of risk stratification is frequency of follow-up. A key study by Nickel et al. [42] in patients with idiopathic PAH demonstrated that follow-up measurements of WHO FC, cardiac index, mixed venous oxygen saturation and NT-proBNP had higher predictive value versus baseline for transplantation-free survival, underlying the importance of assessing these variables throughout the course of disease.

\section{Screening for PAH in systemic sclerosis}

In addition to risk stratification, BNP and NT-proBNP can also be used to screen for PAH in connective tissue disease, especially SSc. A study of 109 patients with SSc and NT-proBNP >395 ng. $\mathrm{L}^{-1}$ showed a positive predictive value of $95.1 \%$ (sensitivity $55.9 \% /$ specificity $95.1 \%$ ) and negative predictive value of $56.5 \%$ for associated PAH, and changes in NT-proBNP levels were highly predictive of mortality (baseline NT-proBNP: HR=4.82; follow-up NT-proBNP: HR=3.82) [25]. In the DETECT study, which proposed a novel algorithm based on ESC/ERS guidelines and including NT-proBNP in multivariable logistic regression models (estimated coefficient (95\% CI) 0.915 (0.308-1.521), p=0.003), 19\% of SSc patients had RHC-confirmed PAH [16]. The Australian Scleroderma Interest Group (ASIG) has also recently incorporated NT-proBNP into a screening algorithm [43].

\section{BNP and NT-proBNP considerations for sampling \\ Physiological characteristics}

RHC is the gold standard for measuring mPAP; however, in clinical practice, the presence of PH is usually first suspected following noninvasive testing. TTE is typically recommended if $\mathrm{PH}$ is suspected and has 
good sensitivity (0.79-1.0) and specificity (0.60-0.98), but can also under- and over-estimate PAP [28]. Imaging investigations such as computed tomography may also suggest the presence of $\mathrm{PH}$ by demonstrating pulmonary artery enlargement or RV dilation amongst other features [11].

Elevated BNP/NT-proBNP can indicate several cardiovascular pathologies including LV dysfunction, LV hypertrophy and PAH [1, 14], although other factors that can influence BNP concentrations must be considered and abnormal levels need to be put into clinical context $[1,44]$. In addition to being indicators of ventricular stress, BNP and NT-proBNP values are also increased by demographic factors such as higher age, female sex and body weight, as well as the presence of chronic renal failure, type 2 diabetes mellitus, anaemia, pulmonary embolism and acute coronary syndrome [45-47]. In renal failure for example, circulating levels of BNP and NT-proBNP inversely correlate with kidney function, though due to the fact that NT-proBNP is exclusively excreted into urine, the effect is much more pronounced for this biomarker [48].

Standardisation of blood draw is not well established. High intra-individual biological variation in patients with stable heart failure has been reported, with reported week-to-week reference change values of $49.2 \%$ and $66.2 \%$ for BNP and NT-proBNP, respectively [49]. Additionally, BRuINs et al. [50] noted that time of day may be a factor, with significant increases of BNP during the day, peaking at $>120 \%$ of the $08: 00 \mathrm{~h}$ baseline at 18:00 h. There is less evidence for significant daily NT-proBNP variation [50, 51].

Consensus is also required on the impact of physical exertion prior to sampling and how to account for this. A systematic review of the effects of running demonstrated that $22.9 \%$ and $35.9 \%$ of individuals who had completed races of varying length and intensity had BNP and NT-proBNP levels above the upper reference limit (typically $100 \mathrm{pg} \cdot \mathrm{mL}^{-1}$ for $\mathrm{BNP}$, which is the recommended acute heart failure threshold [52], and $125 \mathrm{pg} \cdot \mathrm{mL}^{-1}$ for NT-proBNP), respectively, when measured <24 h after an event [53]. Rapid, transient plasma BNP increases well below $100 \mathrm{ng} \cdot \mathrm{L}^{-1}$ have also been observed in healthy individuals undertaking short-term maximal exercise, peaking at a mean $30.6 \pm 4.7 \mathrm{ng} \cdot \mathrm{L}^{-1}$ from a resting level of $19.4 \pm 2.5 \mathrm{ng} \cdot \mathrm{L}^{-1}$ immediately after exercise and returning to baseline within $1 \mathrm{~h} \mathrm{[54]}$.

In cardiovascular disease, small BNP increases in patients after 1 day of $30-50 \mathrm{~km}$ walking exercise (median $28.1 \mathrm{pg} \cdot \mathrm{mL}^{-1}$, baseline to $35.7 \mathrm{pg} \cdot \mathrm{mL}^{-1}$ ) have been reported, though reported baseline levels were already high relative to individuals with cardiovascular risk factors $\left(3.9 \mathrm{pg} \cdot \mathrm{mL}^{-1}\right)$ and healthy controls (5.5 pg. $\mathrm{mL}^{-1}$ ) [55]. Although this is still well below the heart failure threshold, the impact of such increases has not been assessed in patients in PAH where it could conceivably impact on how patients are risk stratified. Similarly, elevated baseline and post-exercise BNP values are reported in individuals with severe LV diastolic dysfunction [56], but not in those with preserved LV ejection fraction [57]. Increases in BNP related to increased physical exertion were also substantially greater compared with individuals with normal diastolic function (96.9 $\mathrm{pg} \cdot \mathrm{mL}^{-1}$ increase versus $12.4 \mathrm{pg} \cdot \mathrm{mL}^{-1}$ ) [56].

Decreases in NT-proBNP with endurance exercise, but increases with strength exercise, have been described in sedentary individuals [58]. However, KuTSCH et al. [59] in a study of 63 patients, concluded that accounting for exercise-related changes in NT-proBNP did not improve correlation with other surrogate markers of disease.

\section{Laboratory assays}

Clinical laboratory assays have several limitations that can potentially impact the efficacy of patient monitoring [60]. Analysis protocols involve multiple stages, including specific pre-analytical processes that, combined with resource or capacity restrictions in a hospital, mean that time-to-results can take up to several days. This limits the potential use of BNP in the emergency setting, as well as "BNP-guided therapy", where serial measurements could be used to monitor patients and potentially guide therapy. For example, the need for specialist laboratories prevents monitoring in primary care or at the patient's home, an approach which has been demonstrated to be potentially useful in early detection of cardiac decompensation in high-risk patients in a study of 163 patients with signs and symptoms of acute heart failure [61].

There is no standard protocol for BNP or NT-proBNP sampling and testing. Although risk stratification guidelines recommend specific threshold values to indicate PAH severity (table 1), accuracy and analytical range can vary between tests, and there is conflicting evidence on interchangeability of results. Despite this, studies confirm the ability of BNP and NT-proBNP to independently predict cardiovascular mortality across different thresholds, time intervals and prognostic models [15].

Poor diagnostic concordance between the two biomarkers for ruling in/out heart failure has also been reported, with highly variable levels of the two peptides over time [62]. This can be exacerbated by chronic kidney disease, in part due to elevation of NT-proBNP relative to BNP in these patients. Another study by 
TABLE 1 Comparison of European Society of Cardiology (ESC)/European Respiratory Society (ERS) and REVEAL 2.0 prognostic tools

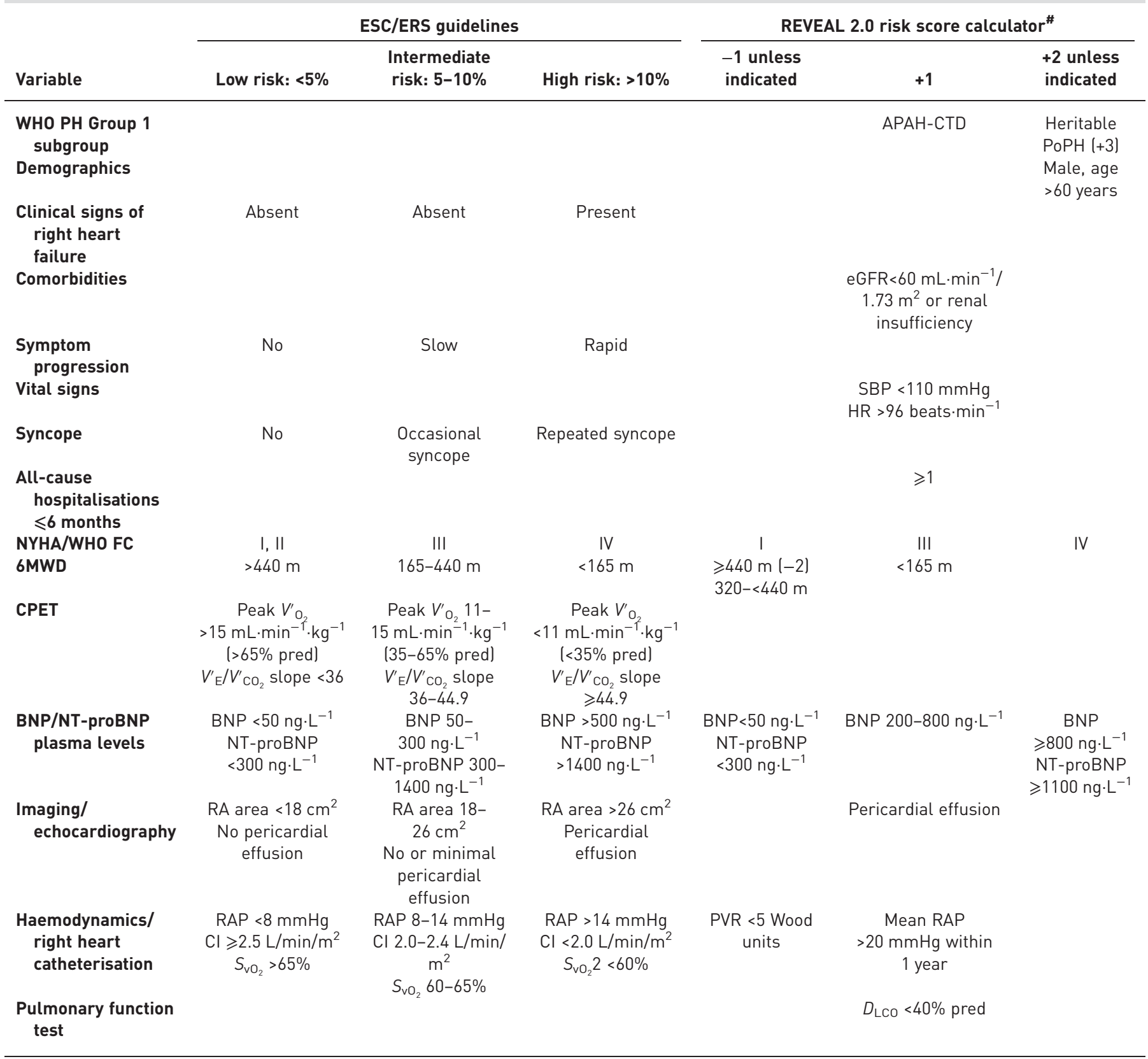

ESC/ERS guidelines state that, while not all variables need to be assessed, WHO FC and at least one measurement of exercise capacity (6MWD or CPET) should be taken as a minimum, and assessment of right ventricular (RV) function (BNP/NT-proBNP or echocardiography) is recommended. REVEAL 2.0 includes 11 variables including modifiable and non-modifiable with each score corresponding to a risk for mortality at 1 year. To mirror the ESC/ERS approach the REVEAL score can also be split into three groups llow risk $\leqslant 6$, intermediate risk 7-8, high risk 29] [1, 9]. REVEAL: Registry to Evaluate Early And Long-term PAH Disease Management; WHO: World Health Organization; PH: pulmonary hypertension; NYHA: New York Heart Association; FC: functional class; CPET: cardiopulmonary exercise test; 6MWD: 6-min walk distance; BNP: B-type natriuretic peptide; NT-proBNP: N-terminal pro-hormone of BNP; APAH-CTD: pulmonary arterial hypertension associated with connective tissue disease; PoPH: portopulmonary hypertension; eGFR: estimated glomerular filtration rate; SBP: systolic blood pressure; HR: heart rate; $V^{\prime}{ }_{\mathrm{O}_{2}}$ : oxygen consumption; $V_{E}^{\prime}$ : minute ventilation; $V^{\prime} \mathrm{CO}_{2}$ : carbon dioxide production; RA: right atrium; RAP: right atrial pressure; $\mathrm{Cl}$ : cardiac index; $S_{\mathrm{VO}_{2}}$ : mixed venous oxygen saturation; PVR: pulmonary vascular resistance; $D_{\mathrm{Lco}}$ : diffusing capacity of the lungs for carbon monoxide. " : add/subtract for each variable to get overall score.

Collin-Chavagnac et al. [63] compared 10 different clinical laboratory and POCT assays, reporting that median BNP varied between 315 and $526 \mathrm{ng} \cdot \mathrm{L}^{-1}$ and NT-proBNP between 1020 and $1450 \mathrm{ng} \cdot \mathrm{L}^{-1}$. The authors concluded that, while useful diagnostically, none of the tests could be reliably cross compared and 
recommended that patients should consistently use the same device model [63]. A UK-based consensus group set up to develop clinical guidance in $\mathrm{PAH}$ also recommend users take part in a quality assurance scheme and stick to manufacturer recommendations, given the potential for variation between kits [64].

\section{Comparison of BNP and NT-proBNP as biomarkers in the clinical setting}

BNP and NT-proBNP are both widely used in PAH risk stratification, and the ESC/ERS guidelines state that there is no clear advantage of one over the other [1]. A meta-analysis incorporating 48 evaluations of five different assay products in 37 unique cohorts (BNP: 26 cohorts; NT-proBNP: 18 cohorts) found both molecules achieved "excellent" predictive value for excluding acute heart failure at their lower cut-off thresholds [65]. Similar findings have also been reported specifically in POCT assays, with close correlation in regression analysis $(r=0.93 ; \mathrm{p}<0.01)$ in a single study including 151 patients with structural heart disease [66]. However, there are clinically relevant differences. The advantages and disadvantages of these two molecules for PAH risk stratification are summarised in table 2.

BNP has been shown to correlate better with pulmonary haemodynamics in patients with renal dysfunction as, unlike NT-proBNP, it is captured or cleared by natriuretic peptide receptors or inactivated by neprilysin [1, 48, 67]. Conversely, NT-proBNP is considered more accurate for prognosis and predicting mortality because it integrates renal insufficiency and haemodynamic impairment [1, 67], especially in females and younger patients [68].

In clinical laboratory testing, the longer half-life of NT-proBNP may be beneficial if sample transportation time is high. Estimates of BNP stability recommend that it should be analysed or frozen within $4 \mathrm{~h}$, whereas NT-proBNP can reasonably be stored at room temperature for up to 2 days $[18,64,69]$ Use of POCT, however, eliminates this issue. Another potential advantage of NT-proBNP testing over BNP testing is that all commercially available clinical laboratory immunoassays at time of publication, including POCT assays, are based on the same antibodies and calibrators distributed by Roche Diagnostics (Rotkreuz, Switzerland), making assays relatively consistent [20]. By contrast, BNP immunoassays are diverse, using different antibodies and standard materials. The CardioOrmoCheck study, which distributed 72 study samples to 130 Italian laboratories, found up to $50 \%$ difference in reported BNP values between assays [20, 70, 71], and these inconsistencies remain when using the same antibodies on different instruments $[20,70,72]$. This variety in sensitivity/specificity is also present in studies specifically evaluating BNP POCT [73].

Cross-reactivity with proBNP-derived peptides can affect BNP and NT-proBNP differently between assays. The entire BNP 1-32 peptide remains intact in the C-terminal portion of proBNP, meaning that unprocessed proBNP-108 and O-glycosylated proBNP-108 can be immunoreactive in BNP assays [74-76], potentially elevating reported values. Conversely, the Roche anti-NT-proBNP assay monoclonal antibodies are specific to one region of the molecule (epitope 42-46) which includes a serine at position 44 that can be glycosylated during normal post-translational processing. This can potentially reduce ligand binding

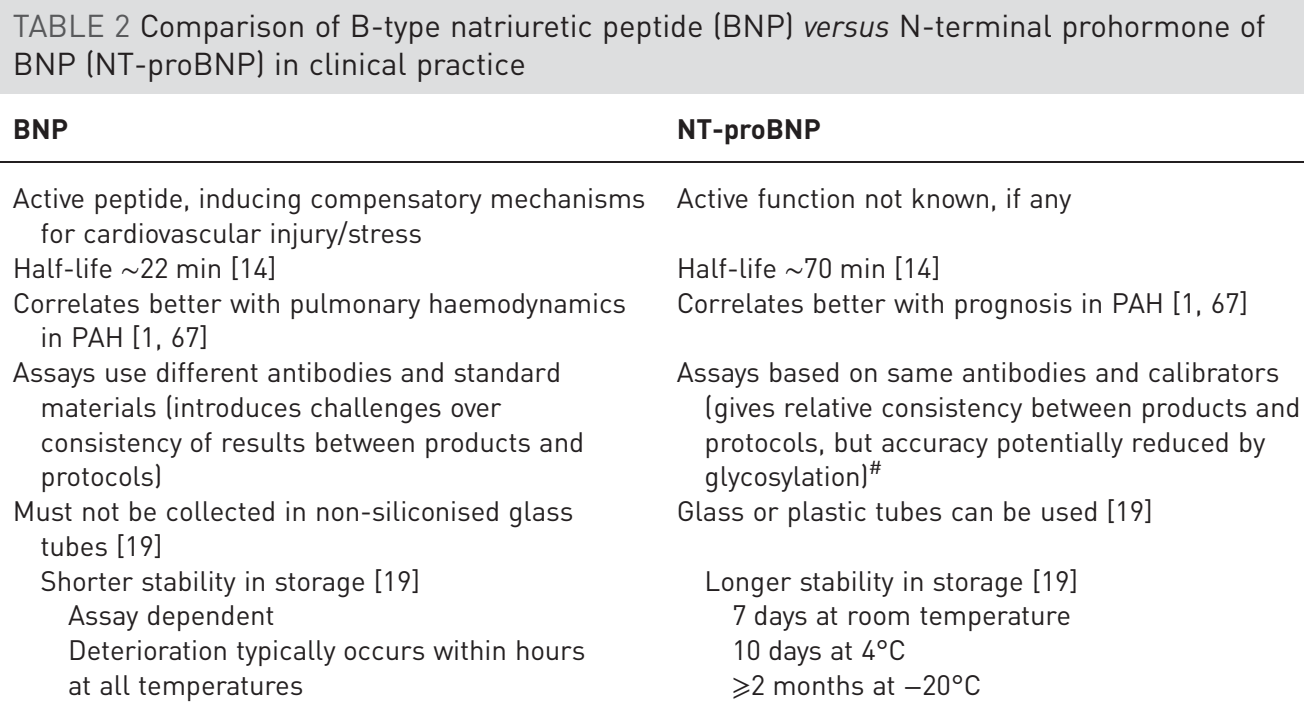

$\mathrm{PAH}$ : pulmonary arterial hypertension. " : extent of glycosylation may be influenced by pathology, e.g. increases seen in chronic renal failure, which would underestimate the true NT-proBNP level. 
and underestimate the true NT-proBNP concentration $[22,72,77,78]$. It has been suggested that extent of glycosylation at this site is influenced by pathology, increased glycosylation seen in patients with heart failure and chronic renal failure on haemodialysis [74, 79]. Pre-treating samples with deglycosylation enzymes has been shown to ameliorate these diagnostic limitations $[79,80]$, although further validation studies are required before clinical adoption.

While standardisation for BNP and NT-proBNP assays is technically difficult with current technologies, it is feasible that consensus could be reached on a tolerable level of discrepancy [20,72].

\section{Point-of-care testing: what opportunities are there to improve patient outcomes in} patients with PAH?

Between June and December 2019, we carried out PubMed searches for terms and combinations including "pulmonary arterial hypertension", "BNP", "NT-proBNP" and "point-of-care testing", with no restrictions on publication date. While a number of results were found in comparable searches in chronic heart failure [73] and in unexplained dyspnoea, no relevant papers specifically on the subject of POCT in PAH were identified. Despite this, experience from other therapy areas suggests that POCT has the potential to provide a number of benefits including reduced time-to-result and time-to-diagnosis, and ease of handling, together with similar analytical performance, which may lead to improved patient outcomes and clinical cost effectiveness.

\section{Time-to-result}

One of the potential benefits of BNP/NT-proBNP POCT is improved time-to-result, which typically takes 8-20 min depending on the device, whereas clinical laboratory results are typically turned around in excess of $24 \mathrm{~h}$, although at their quickest, dedicated emergency room STAT laboratories can run tests in $<1 \mathrm{~h}$ [81]. BNP POCT assays are well established in other areas, especially in patients with unexplained breathlessness and screening for heart failure, and have been used clinically throughout the last two decades. A 2001 study by MaIsel et al. [82] describes how in 200 individuals being evaluated for LV dysfunction, POCT could reliably predict diagnosis by echocardiogram, with an area under the curve of 0.95 and $98 \%$ specificity at a BNP level of $75 \mathrm{pg} \cdot \mathrm{mL}^{-1}$.

\section{Analytical performance}

The analytical performance of POCT is typically described as either comparable to or slightly lower than clinical laboratory assays, but there is general agreement that POCT is reliable enough to be used as a management tool $[60,83,84]$. In patients with LV dysfunction, for example, there are multiple studies to show POCT assays are comparable to clinical laboratory assays when using the same antibodies and materials $[85,86]$. Device-specific studies of BNP/NT-proBNP POCT include: Triage BNP (Quidel; San Diego, CA, USA) [87, 88]; RAMP NT-proBNP (Response Biomedical; Vancouver, Canada) [89]; cobas h232 (Roche Diagnostics) [90-92]; Minicare BNP (Philips; Eindhoven, The Netherlands) [93]; i-STAT (Abbott; Princeton, NJ, USA), which studies noted had good agreement with clinical laboratory despite low precision [88, 94]; PATHFAST NT-proBNP (Mitsubishi Chemical Europe; Düsseldorf, Germany) [95, 96]; AQT90 FLEX (Radiometer; Copenhagen, Denmark) [97]; Alere NT-proBNP (now Quidel) [98] and Alere Heart Check, although lower precision than clinical laboratory was noted [60, 84, 99]; Rapidpia (Sekisui Medical Co.; Tokyo, Japan) [100]; and SHIONOSPOT (Shionogi \& Co.; Osaka, Japan) [100] (table 3). POCT assays also typically have a narrower analytical range than clinical laboratory assays, and this may limit their utility in patients with extreme values [66].

\section{Time-to-diagnosis}

In clinical practice, the turnaround time offered by POCT could potentially translate into improved patient outcomes through reducing time-to-diagnosis/prognosis and treatment, especially in the emergency setting when diagnosis is unclear [81]. In one centre that introduced POCT for a number of biomarker assays, time from sample collection to reporting in the emergency department reduced from 70 to 24 min [101]. In BNP POCT specifically, one of the largest trials of its kind (1586 patients), demonstrated that the Biosite (now Quidel) Triage BNP kit was reliable enough for use in the emergency room, proving more accurate than patient history, physical findings or clinical laboratory assays for identifying the cause of dyspnoea [87]. It also performed better than the National Health and Nutrition Examination Survey or Framingham criteria for diagnosis of chronic heart failure [87].

The BASEL study of dyspnoea also confirmed that, while there was conflicting evidence on the cost benefits of BNP POCT in primary care, it did reduce hospital admission rates and hospital stay duration, improved diagnostic certainty and reduced time-to-diagnosis in the emergency room to 63 min versus 90 min compared with clinical laboratory assays [86, 102-105]. Another study implementing NT-proBNP and troponin POCT in a single centre reported improved patient outcomes in acute coronary syndrome 
TABLE 3 Comparison of 10 currently available BNP/NT-proBNP POCT devices

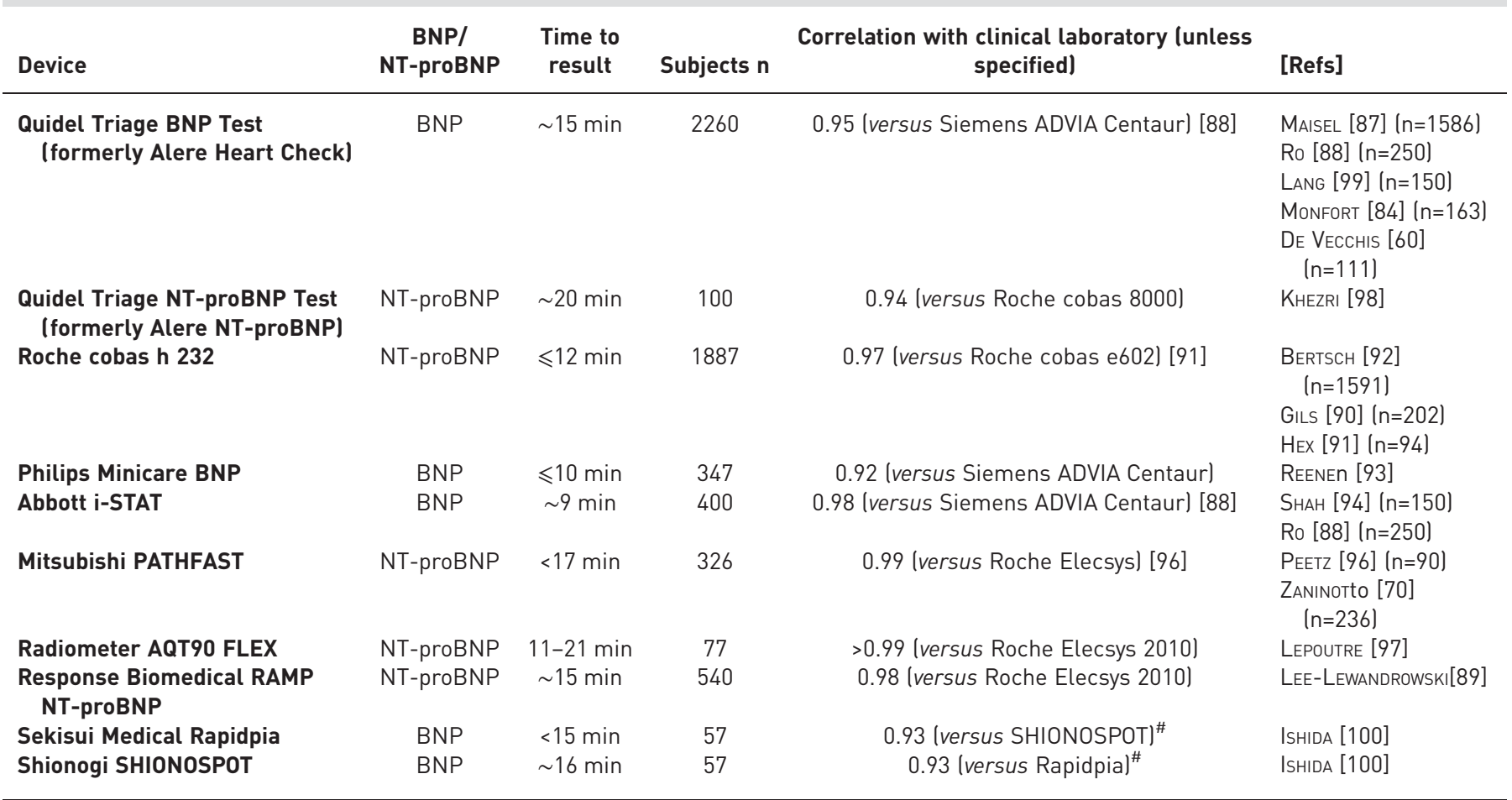

BNP: B-type natriuretic peptide; NT-proBNP: N-terminal prohormone of BNP; POCT: point-of-care testing. ${ }^{\#}$ : Sekisui Medical Rapidpia and Shionogi SHIONOSPOT are both POCT devices compared with each other in this study.

compared with similar hospitals, reducing 30-day readmission rates from $10.4 \%$ to $4.2 \%$, and hospital mortality from $15.8 \%$ to $9.8 \%$. The authors concluded that "POCT has been critical to the success of the network, but it needs to be implemented within an integrated system of care to produce optimal outcomes" [106].

\section{Ease of handling}

POCT also potentially reduces handling errors by being easier to use and simpler to implement than clinical laboratory assays. Several steps in the clinical laboratory testing pathway can be eliminated, including transport, storage, pre-analytical processing, result validation and return of results to treating clinician, as well as the requirement for multiple patient consultations [107].

Multiple studies have determined that POCT can be used by physicians at all levels, as well as by allied healthcare professionals and by patients/caregivers. One study found that with at least two standardised training sessions $(\sim 1.5 \mathrm{~h}$ total), GPs were successfully taught to use BNP POCT for heart failure, which improved clinical decision making [108]. Another "untrained user study" using the Triage BNP device gave only standard user instructions to operators, who reported comparable values to clinicians experienced with the same device [83].

The HABIT study trained patients with heart failure symptoms to measure BNP at home from blood samples. Of note, the ability to obtain daily data also revealed insights into the natural physiology of BNP which fluctuated on a day-to-day basis in specific patients [61]. A further study into patient use of POCT in heart failure achieved measurements that correlated well with those taken by healthcare professionals [99].

\section{Reduced costs}

Although POCT usually has higher initial per-test costs than clinical laboratory assays, net savings can result from reduced time-to-diagnosis/treatment and reduced hospitalisation time [107]. For example, despite slightly lower accuracy, BNP/NT-proBNP POCT was demonstrated to be reliable enough as a prognostic indicator [28], so could potentially lower costs by reducing the need for echocardiography. SIEBERT et al. [109] also report that NT-proBNP testing could reduce echocardiography use by up to 58\%, reducing overall per patient costs by $9.4 \%$. In this study, shorter time-to-diagnosis also resulted in a modest decrease in serious adverse event rates and patient hospital days. Additionally, a Spanish centre 
identified an optimal threshold of $280 \mathrm{ng} \cdot \mathrm{L}^{-1}$ using the cobas h232 POCT device to rule out heart failure and subsequently reduced the need for echocardiography by $67 \%$ [110].

On a national scale, an NHS audit reported that replacing echocardiography where possible with NT-proBNP testing throughout the UK could save $£ 1.6$ million per year [111]. A Norwegian study similarly reported that the 1-year societal costs of heart failure were lowest when NT-proBNP POCT was used (€505) compared with diagnosis by history/clinical findings (€543) and clinical laboratory (€607), with the savings due to fewer GP visits and less use of spirometry [112].

However, several studies have failed to identify a significant benefit to POCT, although they also did not conclude that POCT is disadvantageous compared with clinical laboratory testing. In a study of 711 patients seen in dyspnoea triage, it was found that NT-proBNP POCT made no difference to time in hospital, intensive care unit admission rates or mortality [113]. A systematic review in diagnostic accuracy for acute cardiopulmonary symptoms reported limited and inconclusive evidence that GP use of POCT leads to more accurate diagnosis and improvements to clinical management [114].

\section{What do we need to know before introducing POCT to PAH assessment?}

In theory, currently available and well-established POCT devices would be applicable to PAH risk stratification, but clinical evidence of their use in this therapy area is lacking.

In heart failure, diagnosis is typically a binary result that includes/excludes pathology depending on BNP/ NT-proBNP thresholds, though borderline values may still require interpretation or further testing. Risk stratification can be more subtle with multiple thresholds (e.g. low, medium or high) and requiring multiple factors to be taken into account. As a consequence, more research is required to determine if the reported lower accuracy of POCT assays may impact on their use in PAH?

The commonly used threshold values in heart failure due to LV dysfunction presention are $35 \mathrm{ng} \cdot \mathrm{L}^{-1}$ for BNP and $125 \mathrm{ng} \cdot \mathrm{L}^{-1}$ for NT-proBNP, or $100 \mathrm{ng} \cdot \mathrm{L}^{-1}$ and $300 \mathrm{ng} \cdot \mathrm{L}^{-1}$, respectively, in the acute setting [52]. High sensitivity of both clinical and POCT BNP assays at $\sim 100 \mathrm{ng} \cdot \mathrm{L}^{-1}$ has been reported [115]. In PAH risk stratification, the ESC/ERS guideline threshold values are higher: BNP: low $<50 \mathrm{ng} \cdot \mathrm{L}^{-1}$, intermediate 50-300 ng. $\mathrm{L}^{-1}$, high >300 ng. $\mathrm{L}^{-1}$; NT-proBNP: low <300 ng. $\mathrm{L}^{-1}$, intermediate $300-1400 \mathrm{ng} \cdot \mathrm{L}^{-1}$, high $>1400 \mathrm{ng} \cdot \mathrm{L}^{-1}$ [1]. REVEAL 2.0 has similar thresholds (BNP: low $<50 \mathrm{ng} \cdot \mathrm{L}^{-1}$, intermediate $200-800 \mathrm{ng} \cdot \mathrm{L}^{-1}$, high $\geqslant 800 \mathrm{ng} \cdot \mathrm{L}^{-1}$; NT-proBNP: low $<300 \mathrm{ng} \cdot \mathrm{L}^{-1}$, high $\geqslant 1100 \mathrm{ng} \cdot \mathrm{L}^{-1}$ ) [9], though both scores are comfortably within the analytical range of available POCT devices [60, 84, 87-100, 116].

Capillary samples have been shown to offer good correlation with venous samples in several studies ranging from 111 to 187 patients each [60,93], as well as acceptable reproducibility [117]. Further research is required to identify whether the specific pathophysiology of $\mathrm{PAH}$ affects the synthesis and secretion of biomarker molecules, and hence the reliability of BNP/NT-proBNP as a biomarker in capillary samples, especially in the context of physical exertion before sampling.

There is also the question of whether the rapid availability of results in the PAH clinic offers a similar level of added value to that seen in other pathologies. Daily, self-administered BNP POCT has been shown to be feasible and safe in outpatients. In the HABIT Trial, daily BNP testing was performed and correlated with adverse outcomes and was complementary to weight monitoring [61]. The value of such regular monitoring in PAH and whether it could be used to direct treatment and guide diuretic therapy is not known.

\section{Conclusion}

BNP/NT-proBNP POCT has been successfully implemented and is well established in multiple cardiovascular pathologies, including acute and chronic heart failure due to left ventricular dysfunction and dyspnoea. In the majority of studies, POCT has been shown to confer benefits including improved patient outcomes through reduced time-to-diagnosis and cost effectiveness. No studies currently exist in $\mathrm{PAH}$ using POCT and further work is required to assess its potential clinical utility.

A key challenge in BNP/NT-proBNP testing, whether clinical laboratory or POCT, is the lack of standardisation between protocols and devices. While POCT provides opportunities to simplify clinical measurement of these biomarkers, more research is needed to standardise approaches to sampling and assess their clinical utility.

Acknowledgements: Writing assistance was provided by Simon Lott (Connect2 CME Ltd, Westerham, UK).

Conflict of interest: R.A. Lewis reports non-financial support from Actelion Pharmaceuticals, outside the submitted work. C. Durrington has nothing to disclose. R. Condliffe reports personal fees and non-financial support from Actelion Pharmaceuticals, Bayer, GlaxoSmithKline and Merck Sharp \& Dohme, outside the submitted work. D.G. Kiely reports 
personal fees and non-financial support from Actelion Pharmaceuticals, Bayer, GlaxoSmithKline and Merck Sharp \& Dohme, outside the submitted work.

Support statement: This work was funded by a grant from Janssen Ltd awarded to support manuscript writing. Funding information for this article has been deposited with the Crossref Funder Registry.

\section{References}

1 Galie N, Humbert M, Vachiery JL, et al. 2015 ESC/ERS Guidelines for the diagnosis and treatment of pulmonary hypertension: The Joint Task Force for the Diagnosis and Treatment of Pulmonary Hypertension of the European Society of Cardiology (ESC) and the European Respiratory Society (ERS): Endorsed by: Association for European Paediatric and Congenital Cardiology (AEPC), International Society for Heart and Lung Transplantation (ISHLT). Eur Heart J 2016; 37: 67-119.

2 Hoeper MM, Kramer T, Pan Z, et al. Mortality in pulmonary arterial hypertension: prediction by the 2015 European pulmonary hypertension guidelines risk stratification model. Eur Respir J 2017; 50: 1700740.

3 Simonneau G, Galie N, Rubin LJ, et al. Clinical classification of pulmonary hypertension. J Am Coll Cardiol 2004; 43: Suppl. S12, 5S-12S.

4 Simonneau G, Montani D, Celermajer DS, et al. Haemodynamic definitions and updated clinical classification of pulmonary hypertension. Eur Respir J 2019; 53: 1801913.

5 Lai YC, Potoka KC, Champion HC, et al. Pulmonary arterial hypertension: the clinical syndrome. Circ Res 2014; 115: $115-130$

6 Kiely DG, Elliot CA, Sabroe I, et al. Pulmonary hypertension: diagnosis and management. BMJ 2013; 346: f2028.

7 Hoeper M, Gibbs J. The changing landscape of pulmonary arterial hypertension and implications for patient care. Eur Respir Rev 2014; 23: 450-457.

8 Lau EMT, Giannoulatou E, Celermajer DS, et al. Epidemiology and treatment of pulmonary arterial hypertension. Nat Rev Cardiol 2017; 14: 603-614.

9 Benza RL, Gomberg-Maitland M, Elliott CG, et al. Predicting survival in patients with pulmonary arterial hypertension: the REVEAL risk score calculator 2.0 and comparison with ESC/ERS-based risk assessment strategies. Chest 2019; 156: 323-337.

10 Galie N, Channick RN, Frantz RP, et al. Risk stratification and medical therapy of pulmonary arterial hypertension. Eur Respir J 2019; 53: 1801889.

11 Kiely DG, Levin D, Hassoun P, et al. EXPRESS: statement on imaging and pulmonary hypertension from the Pulmonary Vascular Research Institute (PVRI). Pulm Circ 2019; 9: 2045894019841990.

12 Swift AJ, Capener D, Johns C, et al. Magnetic resonance imaging in the prognostic evaluation of patients with pulmonary arterial hypertension. Am J Respir Crit Care Med 2017; 196: 228-239.

13 Lewis RA, Johns CS, Cogliano M, et al. Identification of cardiac MRI thresholds for risk stratification in pulmonary arterial hypertension. Am J Respir Crit Care Med 2020; 201: 458-468.

14 Fu S, Ping P, Wang F, et al. Synthesis, secretion, function, metabolism and application of natriuretic peptides in heart failure. J Biol Eng 2018; 12: 2.

15 Santaguida PL, Don-Wauchope AC, Oremus M, et al. BNP and NT-proBNP as prognostic markers in persons with acute decompensated heart failure: a systematic review. Heart Fail Rev 2014; 19: 453-470.

16 Coghlan JG, Denton CP, Grunig E, et al. Evidence-based detection of pulmonary arterial hypertension in systemic sclerosis: the DETECT study. Ann Rheum Dis 2014; 73: 1340-1349.

17 Potter LR. Natriuretic peptide metabolism, clearance and degradation. FEBS J 2011; 278: 1808-1817.

18 Buckley M, Marcus N, Yacoub M, et al. Prolonged stability of brain natriuretic peptide: importance for non-invasive assessment of cardiac function in clinical practice. Clin Sci 1998; 95: 235-239.

19 Ordonez-Llanos J, Collinson PO, Christenson RH. Amino-terminal pro-B-type natriuretic peptide: analytic considerations. Am J Cardiol 2008; 101: 9-15.

20 Semenov A, Katrukha A. Analytical issues with natriuretic peptides - has this been overly simplified? EJIFCC 2016; 27: 189-207.

21 Casserly B, Klinger J. Brain natriuretic peptide in pulmonary arterial hypertension: biomarker and potential therapeutic agent. Drug Des Devel Ther 2009; 3: 269-287.

22 Fu S, Ping $\mathrm{P}$, Zhu Q, et al. Brain natriuretic peptide and its biochemical, analytical, and clinical issues in heart failure: a narrative review. Front Physiol 2018; 9: 692.

23 Semenov AG, Tamm NN, Seferian KR, et al. Processing of pro-B-type natriuretic peptide: furin and corin as candidate convertases. Clin Chem 2010; 56: 1166-1176.

24 Chin KM, Channick RN, Kim NH, et al. Central venous blood oxygen saturation monitoring in patients with chronic pulmonary arterial hypertension treated with continuous IV epoprostenol: correlation with measurements of hemodynamics and plasma brain natriuretic peptide levels. Chest 2007; 132: 786-792.

25 Williams MH, Handler CE, Akram R, et al. Role of N-terminal brain natriuretic peptide (N-TproBNP) in scleroderma-associated pulmonary arterial hypertension. Eur Heart J 2006; 27: 1485-1494.

26 Gan CT, McCann GP, Marcus JT, et al. NT-proBNP reflects right ventricular structure and function in pulmonary hypertension. Eur Respir J 2006; 28: 1190-1194.

27 Troisi F, Greco S, Brunetti ND, et al. Right heart dysfunction assessed with echography, B-type natriuretic peptide and cardiopulmonary test in patients with chronic heart failure. J Cardiovasc Med (Hagerstown) 2008; 9: 672-676.

28 Goto K, Arai M, Watanabe A, et al. Utility of echocardiography versus BNP level for the prediction of pulmonary arterial pressure in patients with pulmonary arterial hypertension. Int Heart J 2010; 51: 343-347.

29 Helgeson SA, Imam JS, Moss JE, et al. Comparison of brain natriuretic peptide levels to simultaneously obtained right heart hemodynamics in stable outpatients with pulmonary arterial hypertension. Diseases 2018; 6: E33.

30 Fenster BE, Lasalvia L, Schroeder JD, et al. Cystatin C: a potential biomarker for pulmonary arterial hypertension. Respirology 2014; 19: 583-589.

31 Blyth KG, Groenning BA, Mark PB, et al. NT-proBNP can be used to detect right ventricular systolic dysfunction in pulmonary hypertension. Eur Respir J 2007; 29: 737-744. 
Tulevski I, Groenink M, van der Wall EE, et al. Increased brain and atrial natriuretic peptides in patients with chronic right ventricular pressure overload: correlation between plasma neurohormones and right ventricular dysfunction. Heart 2001; 86: 27-30.

33 Leuchte $\mathrm{HH}$, Ten Freyhaus $\mathrm{H}$, Gall H, et al. Risk stratification strategy and assessment of disease progression in patients with pulmonary arterial hypertension: updated recommendations from the Cologne Consensus Conference 2018. Int J Cardiol 2018; 272S: 20-29.

34 Kylhammar D, Kjellstrom B, Hjalmarsson C, et al. A comprehensive risk stratification at early follow-up determines prognosis in pulmonary arterial hypertension. Eur Heart J 2018; 39: 4175-4181.

35 Boucly A, Weatherald J, Savale L, et al. Risk assessment, prognosis and guideline implementation in pulmonary arterial hypertension. Eur Respir J 2017; 50: 1700889.

36 Weatherald J, Boucly A, Launay D, et al. Haemodynamics and serial risk assessment in systemic sclerosis associated pulmonary arterial hypertension. Eur Respir J 2018; 52: 1800678.

37 Sitbon O, Chin KM, Channick RN, et al. Risk assessment in pulmonary arterial hypertension: Insights from the GRIPHON study. J Heart Lung Transplant 2020; 39: 300-309.

38 Benza RL, Miller DP, Gomberg-Maitland M, et al. Predicting survival in pulmonary arterial hypertension: insights from the Registry to Evaluate Early and Long-Term Pulmonary Arterial Hypertension Disease Management (REVEAL). Circulation 2010; 122: 164-172.

39 Benza RL, Miller DP, Foreman AJ, et al. Prognostic implications of serial risk score assessments in patients with pulmonary arterial hypertension: a Registry to Evaluate Early and Long-Term Pulmonary Arterial Hypertension Disease Management (REVEAL) analysis. J Heart Lung Transplant 2015; 34: 356-361.

40 McGoon MD, Krichman A, Farber HW, et al. Design of the REVEAL Registry for US patients with pulmonary arterial hypertension. Mayo Clin Proc 2008; 83: 923-931.

41 Anderson JJ, Lau EM, Lavender M, et al. Retrospective validation of the REVEAL 2.0 risk score with the Australian and New Zealand Pulmonary Hypertension Registry Cohort. Chest 2020; 157: 162-172.

42 Nickel N, Golpon H, Greer M, et al. The prognostic impact of follow-up assessments in patients with idiopathic pulmonary arterial hypertension. Eur Respir J 2012; 39: 589-596.

43 Quinlivan A, Proudman S, Sahhar J, et al. Cost savings with a novel algorithm for early detection of systemic sclerosis-related pulmonary arterial hypertension: alternative scenario analyses. Intern Med J 2019; 49: $781-785$.

44 Nishimura M, Brann A, Chang KW, et al. The confounding effects of non-cardiac pathologies on the interpretation of cardiac biomarkers. Curr Heart Fail Rep 2018; 15: 239-249.

45 Nayer J, Aggarwal P, Galwankar S. Utility of point-of-care testing of natriuretic peptides (brain natriuretic peptide and n-terminal pro-brain natriuretic peptide) in the emergency department. Int J Crit Illn Inj Sci 2014; 4: $209-215$.

46 Kiely DG, Kennedy NS, Pirzada O, et al. Elevated levels of natriuretic peptides in patients with pulmonary thromboembolism. Respir Med 2005; 99: 1286-1291.

47 Raymond I, Groenning BA, Hildebrandt PR, et al. The influence of age, sex and other variables on the plasma level of N-terminal pro brain natriuretic peptide in a large sample of the general population. Heart 2003; 89: 745-751.

48 Takase H, Dohi Y. Kidney function crucially affects B-type natriuretic peptide (BNP), N-terminal proBNP and their relationship. Eur J Clin Invest 2014; 44: 303-308.

49 O'Hanlon R, O'Shea P, Ledwidge M, et al. The biologic variability of B-type natriuretic peptide and N-terminal pro-B-type natriuretic peptide in stable heart failure patients. J Card Fail 2007; 13: 50-55.

50 Bruins S, Fokkema MR, Romer JW, et al. High intraindividual variation of B-type natriuretic peptide (BNP) and amino-terminal proBNP in patients with stable chronic heart failure. Clin Chem 2004; 50: 2052-2058.

51 Goetze JP, Jørgensen HL, Sennels HP, et al. Diurnal plasma concentrations of natriuretic propeptides in healthy young males. Clin Chem 2012; 58: 789-792.

52 Ponikowski P, Voors AA, Anker SD, et al. 2016 ESC Guidelines for the diagnosis and treatment of acute and chronic heart failure: the Task Force for the diagnosis and treatment of acute and chronic heart failure of the European Society of Cardiology (ESC) developed with the special contribution of the Heart Failure Association (HFA) of the ESC. Eur Heart J 2016; 37: 2129-2200.

53 Vilela EM, Bettencourt-Silva R, Nunes JP, et al. BNP and NT-proBNP elevation after running - a systematic review. Acta Cardiol 2015; 70: 501-509.

54 Krupicka J, Janota T, Kasalová Z, et al. Effect of short-term maximal exercise on BNP plasma levels in healthy individuals. Physiol Res 2010; 2010: 625-628.

55 Aengevaeren VL, Hopman MTE, Thijssen DHJ, et al. Endurance exercise-induced changes in BNP concentrations in cardiovascular patients versus healthy controls. Int J Cardiol 2017; 227: 430-435.

56 Stepien-Walek A, Wozakowska-Kaplon B. The effect of left ventricular diastolic function on the secretion of B-type natriuretic peptide at rest and directly after exercise test in asymptomatic patients with diabetes or after myocardial infarction with preserved left ventricular systolic function. Kardiol Pol 2016; 74: 529-536.

57 Zdrenghea D, Ilea M, Bodizs G, et al. NT-pro-BNP during isotonic and isometric exercise in heart failure patients with preserved LV ejection fraction. Clin Lab 2014; 60: 2055-2061.

58 Bordbar S, Bigi MA, Aslani A, et al. Effect of endurance and strength exercise on release of brain natriuretic peptide. J Cardiovasc Dis Res 2012; 3: 22-25.

59 Kutsch J, Faul C, von Scheidt W, et al. The association of the N-terminal pro-brain-type natriuretic peptide response to exercise with disease severity in therapy-naive pulmonary arterial hypertension: a cohort study. Respir Res 2018; 19: 8.

60 De Vecchis R, Ariano C. Measuring B-type natriuretic peptide from capillary blood or venous sample: is it the same? Cardiol Res 2016; 7: 51-58.

61 Maisel A, Barnard D, Jaski B, et al. Primary results of the HABIT Trial (heart failure assessment with BNP in the home). J Am Coll Cardiol 2013; 61: 1726-1735.

62 Farnsworth CW, Bailey AL, Jaffe AS, et al. Diagnostic concordance between NT-proBNP and BNP for suspected heart failure. Clin Biochem 2018; 59: 50-55.

63 Collin-Chavagnac D, Dehoux M, Schellenberg F, et al. Head-to-head comparison of 10 natriuretic peptide assays. Clin Chem Lab Med 2015; 53: 1825-1837.

64 Cowie M, O'Collinson P, Dargie H, et al. Recommendations on the clinical use of B-type natriuretic peptide testing (BNP or NTproBNP) in the UK and Ireland. Br J Cardiol 2010; 17: 76-80. 
Roberts E, Ludman AJ, Dworzynski K, et al. The diagnostic accuracy of the natriuretic peptides in heart failure: systematic review and diagnostic meta-analysis in the acute care setting. BMJ 2015; 350: h910. established BNP (Triage BNP $^{\circledast}$ ) point-of-care tests. Biomark Med 2012; 6: 789-796.

67 Leuchte H, El Nounou M, Tuerpe J, et al. N-terminal pro-brain natriuretic peptide and renal insufficiency as predictors of mortality in pulmonary hypertension. Chest 2007; 131: 402-409.

68 Kara K, Lehmann N, Neumann T, et al. NT-proBNP is superior to BNP for predicting first cardiovascular events in the general population: the Heinz Nixdorf Recall Study. Int J Cardiol 2015; 183: 155-161.

69 Downie PF, Talwar S, Squire IB, et al. Assessment of the stability of N-terminal pro-brain natriuretic peptide in vitro: implications for assessment of left ventricular dysfunction. Clin Sci 1999; 97: 255-258.

70 Clerico A, Zaninotto M, Prontera C, et al. State of the art of BNP and NT-proBNP immunoassays: the CardioOrmoCheck study. Clin Chim Acta 2012; 414: 112-119.

71 Prontera C, Zaninotto M, Giovannini S, et al. Proficiency testing project for brain natriuretic peptide (BNP) and the N-terminal part of the propeptide of BNP (NT-proBNP) immunoassays: the CardioOrmocheck study. Clin Chem Lab Med 2009; 47: 762-768.

72 Semenov AG, Feygina EE. Standardization of BNP and NT-proBNP immunoassays in light of the diverse and complex nature of circulating BNP-related peptides. Adv Clin Chem 2018; 85: 1-30.

73 Taylor KS, Verbakel JY, Feakins BG, et al. Diagnostic accuracy of point-of-care natriuretic peptide testing for chronic heart failure in ambulatory care: systematic review and meta-analysis. BMJ 2018; 361: k1450.

74 Nishikimi T, Kuwahara K, Nakagawa Y, et al. Complexity of molecular forms of B-type natriuretic peptide in heart failure. Heart 2013; 99: 677-679.

75 Saenger AK, Rodriguez-Fraga O, Ler R, et al. Specificity of B-type natriuretic peptide assays: cross-reactivity with different BNP, NT-proBNP, and proBNP Peptides. Clin Chem 2017; 63: 351-358.

76 Clerico A, Passino C, Franzini M, et al. Cardiac biomarker testing in the clinical laboratory: where do we stand? General overview of the methodology with special emphasis on natriuretic peptides. Clin Chim Acta 2015; 443: $17-24$.

77 Seferian KR, Tamm NN, Semenov AG, et al. Immunodetection of glycosylated NT-proBNP circulating in human blood. Clin Chem 2008; 54: 866-873.

78 Kuwahara K, Nakagawa Y, Nishikimi T. Cutting edge of brain natriuretic peptide (BNP) research - the diversity of BNP immunoreactivity and its clinical relevance. Circ J 2018; 82: 2455-2461.

79 Nishikimi T, Ikeda M, Takeda Y, et al. The effect of glycosylation on plasma N-terminal proBNP-76 levels in patients with heart or renal failure. Heart 2012; 98: 152-161.

80 Røsjø H, Dahl MB, Jorgensen M, et al. Influence of glycosylation on diagnostic and prognostic accuracy of N-terminal pro-B-type natriuretic peptide in acute dyspnea: data from the Akershus Cardiac Examination 2 Study. Clin Chem 2015; 61: 1087-1097.

81 Iwaz JA, Maisel AS. Recent advances in point-of-care testing for natriuretic peptides: potential impact on heart failure diagnosis and management. Expert Rev Mol Diagn 2016; 16: 641-650.

82 Maisel AS, Koon J, Krishnaswamy P, et al. Utility of B-natriuretic peptide as a rapid, point-of-care test for screening patients undergoing echocardiography to determine left ventricular dysfunction. Am Heart J 2001; 141: 367-374.

83 Bingisser R, Cairns CB, Christ M, et al. Measurement of natriuretic peptides at the point of care in the emergency and ambulatory setting: current status and future perspectives. Am Heart J 2013; 166: 614-621.

84 Monfort A, Da Silva K, Vodovar N, et al. Clinical evaluation of the Heart Check system, a new quantitative measurement of fresh capillary BNP. Biomark Med 2015; 9: 1323-1330.

85 Clerico A, Franzini M, Masotti S, et al. State of the art of immunoassay methods for B-type natriuretic peptides: An update. Crit Rev Clin Lab Sci 2015; 52: 56-69.

86 Florkowski C, Don-Wauchope A, Gimenez N, et al. Point-of-care testing (POCT) and evidence-based laboratory medicine (EBLM) - does it leverage any advantage in clinical decision making? Crit Rev Clin Lab Sci 2017; 54: 471-494.

87 Maisel A, Krishnaswamy P, Nowak R, et al. Rapid measurement of B-type natriuretic peptide in the emergency diagnosis of heart failure. $N$ Engl J Med 2002; 347: 161-167.

88 Ro R, Thode HC Jr, Taylor M, et al. Comparison of the diagnostic characteristics of two B-type natriuretic peptide point-of-care devices. J Emerg Med 2011; 41: 661-667.

89 Lee-Lewandrowski E, Januzzi JL, Green SM, et al. Multi-center validation of the response biomedical corporation RAMP NT-proBNP assay with comparison to the Roche Diagnostics GmbH Elecsys proBNP assay. Clin Chim Acta 2007; 386: 20-24.

90 Gils C, Ramanathan R, Breindahl T, et al. NT-proBNP on Cobas h 232 in point-of-care testing: performance in the primary health care versus in the hospital laboratory. Scand J Clin Lab Invest 2015; 75: 602-609.

91 Hex C, Smeets M, Penders J, et al. Accuracy, user-friendliness and usefulness of the Cobas h232 point-of-care test for NT-proBNP in primary care. J Clin Pathol 2018; 71: 539-545.

92 Bertsch T, Chapelle J, Dempfle C, et al. Multicentre analytical evaluation of a new point-of-care system for the determination of cardiac and thromboembolic markers. Clin Lab 2010; 56: 37-49.

93 Reenen AV, Berger M, Moreau E, et al. Analytical performance of a single epitope B-type natriuretic peptide sandwich immunoassay on the Minicare platform for point-of-care diagnostics. Pract Lab Med 2019; 15 : e00119.

94 Shah K, Terracciano G, Jiang K, et al. Comparability of results between point-of-care and automated instruments to measure B-type natriuretic peptide. West J Emerg Med 2010; 11: 44-48.

95 Zaninotto M, Mion MM, Di Serio F, et al. PATHFAST NT-proBNP (N-terminal-pro B type natriuretic peptide): a multicenter evaluation of a new point-of-care assay. Clin Chem Lab Med 2010; 48: 1029-1034.

96 Peetz D, Schweigert R, Jachmann N, et al. Method comparison of cardiac marker assays on PATHFAST, StratusCS, AxSYM, Immulite 2000, triage, elecsys and cardiac reader. Clin Lab 2006; 52: 605-614.

97 Lepoutre T, Rousseau M, Ahn S, et al. Measurement Nt-proBNP circulating concentrations in heart failure patients with a new point-of-care assay. Clin Lab 2013; 59: 831-835.

98 Khezri BS, Carlsson L, Larsson A. Evaluation of the Alere NT-proBNP test for point of care testing. J Clin Lab Anal 2016; 30: 290-292. 

comparison of patient and healthcare professional measurement of BNP. Biomark Med 2014; 8: 791-796. devices of B-type natriuretic peptide. Int Heart J 2012; 53: 320-323.

101 Nørgaard B, Mogensen C. Blood sample tube transporting system versus point of care technology in an emergency department; effect on time from collection to reporting? A randomised trial. Scand J Trauma Resusc Emerg Med 2012; 20: 71.

102 Burri E, Hochholzer K, Arenja N, et al. B-type natriuretic peptide in the evaluation and management of dyspnoea in primary care. J Intern Med 2012; 272: 504-513.

103 Mueller C, Laule-Kilian K, Schindler C, et al. Cost-effectiveness of B-type natriuretic peptide testing in patients with acute dyspnea. Arch Intern Med 2006; 166: 1081-1087.

104 Tomonaga Y, Gutzwiller F, Lüscher T, et al. Diagnostic accuracy of point-of-care testing for acute coronary syndromes, heart failure and thromboembolic events in primary care: a cluster-randomised controlled trial. BMC Fam Pract 2011; 12: 12

105 Mueller C, Scholer A, Laule-Kilian K, et al. Use of B-type natriuretic peptide in the evaluation and management of acute dyspnea. N Engl J Med 2004; 350: 647-654.

106 Tideman P, Simpson P, Tirimacco R. Integrating PoCT into clinical care. Clin Biochem Rev 2010; 31: 99-104.

107 Christenson E, Collinson P, Defilippi C, et al. Heart failure biomarkers at point-of-care: current utilization and future potential. Expert Rev Mol Diagn 2014; 14: 185-197.

108 Morbach C, Buck T, Rost C, et al. Point-of-care B-type natriuretic peptide and portable echocardiography for assessment of patients with suspected heart failure in primary care: rationale and design of the three-part Handheld-BNP program and results of the training study. Clin Res Cardiol 2018; 107: 95-107.

109 Siebert U, Januzzi JJ, Beinfeld M, et al. Cost-effectiveness of using N-terminal pro-brain natriuretic peptide to guide the diagnostic assessment and management of dyspneic patients in the emergency department. Am J Cardiol 2006; 98: 800-805.

110 Verdú JM, Comín-Colet J, Domingo M, et al. Rapid point-of-care NT-proBNP optimal cut-off point for heart failure diagnosis in primary care. Rev Esp Cardiol (Engl Ed) 2012; 65: 613-619.

111 Upadhyaya C, Roberts K, Jenkins G, et al. NT pro-BNP test can save NHS $£ 1.6$ million per year. Heart 2016; 102: Suppl. 6, A10.

112 Bugge C, Sether EM, Pahle A, et al. Diagnosing heart failure with NT-proBNP point-of-care testing: lower costs and better outcomes. A decision analytic study. BJGP Open 2018; 2: bjgpopen $18 \times 101596$.

113 Bøtker MT, Jorgensen MT, Stengaard C, et al. Prehospital triage of patients suffering severe dyspnoea using N-terminal pro-brain natriuretic peptide, the PreBNP trial: a randomised controlled clinical trial. Eur Heart $J$ Acute Cardiovasc Care 2018; 7: 302-310.

114 Schols AMR, Stakenborg JPG, Dinant GJ, et al. Point-of-care testing in primary care patients with acute cardiopulmonary symptoms: a systematic review. Fam Pract 2018; 35: 4-12.

115 Martindale JL, Wakai A, Collins SP, et al. Diagnosing acute heart failure in the emergency department: a systematic review and meta-analysis. Acad Emerg Med 2016; 23: 223-242.

$116 \mathrm{Wu} \mathrm{AH}$, Smith A, Christenson RH, et al. Evaluation of a point-of-care assay for cardiac markers for patients suspected of acute myocardial infarction. Clin Chim Acta 2004; 346: 211-219.

117 Prontera C, Masotti S, Franzini M, et al. Comparison between BNP values measured in capillary blood samples with a POCT method and those measured in plasma venous samples with an automated platform. Clin Chem Lab Med 2015; 53: e125-e127. 